EPJ Web of Conferences 16, 06010 (2011)

DOI: $10.1051 /$ epjconf/20111606010

(C) Owned by the authors, published by EDP Sciences, 2011

\title{
Radial velocity observations of VB10
}

\author{
R. Deshpande ${ }^{1, a}$, E. Martin ${ }^{1,2}$, M.R. Zapatero Osorio², C. del Burgo ${ }^{3}$, \\ F. Rodler ${ }^{4}$ and M.M. Montgomery ${ }^{1}$ \\ ${ }^{1}$ University of Central Florida, Department of Physics, Orlando, FL 32816-2385, USA \\ ${ }^{2}$ Centro de Astrobiologia (CSIC-INTA), Ctra. Ajalvir km 4, 28850 Torrejon de Ardoz, Madrid, \\ Spain \\ ${ }^{3}$ School of Cosmic Physics, Dublin Institute of Advance Studies, Ireland \\ ${ }^{4}$ Instituto de Astrofisica de Canarias, 38025 La Laguna, Tenerife, Spain
}

\begin{abstract}
VB 10 is the smallest star known to harbor a planet according to the recent astrometric study of Pravdo \& Shaklan [1]. Here we present near-infrared (J-band) radial velocity of VB 10 performed from high resolution (R 20,000) spectroscopy (NIRSPEC/KECK II). Our results [2] suggest radial velocity variability with amplitude of $\sim 1 \mathrm{~km} / \mathrm{s}$, a result that is consistent with the presence of a massive planet companion around VB10 as found via long-term astrometric monitoring of the star by Pravdo \& Shaklan. Employing an entirely different technique we verify the results of Pravdo \& Shaklan.
\end{abstract}

\section{INTRODUCTION}

We have undertaken a program of radial velocity monitoring of nearby late M-dwarfs [3] that will complement current optical searches to evaluate models on planet formation. As part of our program, we observed Van Biesbroeck 10 (VB 10) on two occasions using the NIRSPEC instrument on Keck II telescope. Combining observations taken by our group using the same instrumental setup earlier this decade we obtained a total of four epochs for the same star, between 2001 and 2008.

VB10, a M8.0V has been a spectral standard [4] and a radial velocity template star [5] for over a decade. Recent observations employing astrometry [1] suggests a massive planet, $6.4 \mathrm{M}_{\text {jup }}$ orbiting VB10 at a distance of 0.36 A.U with an eccentricity of less than 0.98 .

\section{INSTRUMENTAL SETUP AND DATA REDUCTION}

The observations were carried on KECK II telescope in Hawaii. The survey employed Near-Infrared SPECtrograph (NIRSPEC) [6]. In the echelle mode we selected the J-band filter and an entrance slit width of 0 . " 432 while the length of the slit was 12 ". The echelle angle was set at $\sim 63^{\circ}$ for all observations. All the observations have been carried out in the J-band. For consistency all the data was reduced following the procedure described in Zapatero Osorio et al. [7]

\section{DATA ANALYSIS AND RESULTS}

For computation of radial velocity of VB10 we used the following steps: (1) As 2007 epoch had a very good Signal-to-Noise Ratio, we use it as a radial velocity template; (2) Echelle orders abundant with telluric lines, were cross-dispersed in IRAF. This helped in obtaining a wavelength zero-point shift for

ae-mail: rohit@physics.ucf .edu

This is an Open Access article distributed under the terms of the Creative Commons Attribution-Noncommercial License 3.0, which permits unrestricted use, distribution, and reproduction in any noncommercial medium, provided the original work is properly cited. 
Table 1. Summarizes the observation parameters used in observing VB10 during the four epochs. All observations were carried out in the J-band.

\begin{tabular}{llcc}
\hline MJD & Vr $(\mathrm{km} / \mathrm{s})$ & Relative $\mathrm{V}_{\mathrm{r}}(\mathrm{km} / \mathrm{s})$ & $\mathrm{V}_{\text {shift }}(\mathrm{km} / \mathrm{s})$ \\
\hline 52075.58951 & $35.50 \pm 0.60$ & $1.13 \pm 0.30$ & $-0.08 \pm 0.23$ \\
52215.20560 & $34.45 \pm 0.60$ & $0.08 \pm 0.30$ & $-0.17 \pm 0.22$ \\
52215.24225 & $34.40 \pm 0.60$ & $0.03 \pm 0.30$ & $+0.51 \pm 0.23$ \\
54276.55865 & $34.37 \pm 0.30$ & - & - \\
54675.25669 & $34.00 \pm 0.83$ & $-0.37 \pm 0.53$ & $1.07 \pm 0.24$ \\
\hline
\end{tabular}
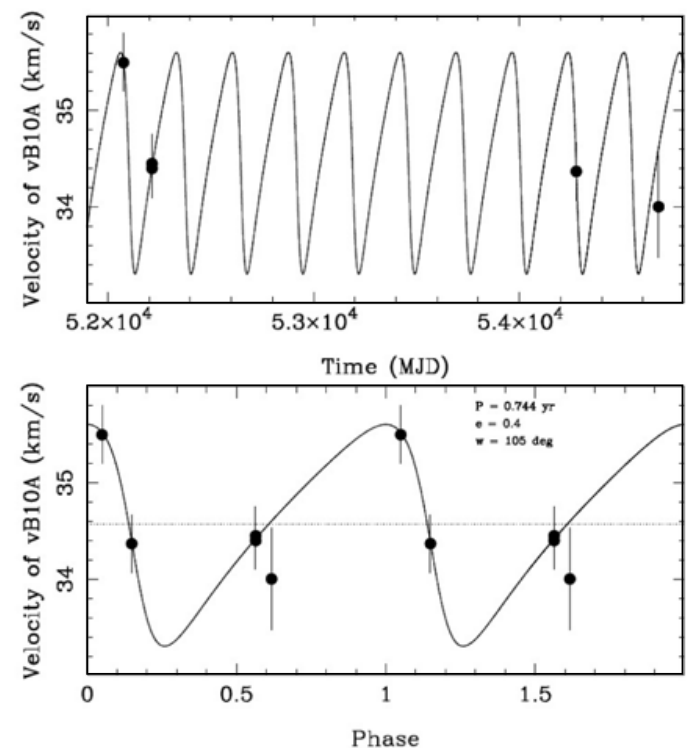

Figure 1. The orbital parameters are noted. Our RV measurements are plotted against MJD (top panel) and the orbital phase (bottom panel). Overplotted on the data is the "most likely" Keplerian solution by Pravdo and Shaklan [1].

the spectra. The values $\left(\mathrm{V}_{\text {shift }}\right)$ are listed in Table 1; (3) Radial velocity measurements $\left(\mathrm{V}_{\mathrm{r}}\right)$ were done on echelle orders that were free of any telluric lines; (4) In order to obtain absolute heliocentric velocity we took into account zero-point shifts errors and corrected for diurnal and annual velocities. The second column in Table 1 shows heliocentric radial velocities for all epochs; (5) the absolute radial velocity was calculated independently using a theoretical procedure followed by del Burgo et al. [8]. The value of $34.05 \pm 0.30 \mathrm{~km} / \mathrm{s}$ obtained through these calculations compares well with those from the table (see Table 1 in del Burgo et al. in this proceedings); (6) The table suggests radial velocity variability with an amplitude of $1 \mathrm{~km} / \mathrm{s}$. Comparing to mean velocity of all measurements, this amplitude is estimated at 3.2- $\sigma$. The variability could be caused due to stellar activity, uncontrolled systematic error in the measurements, or the presence of a massive planet. Although the probability of observing VB10 during its flaring phase is small $(\sim 5 \%)$ with only four epochs of observations, we cannot rule out such a possibility.

From our data points we find orbital eccentricity, e $\leq 0.8$ and periastron (w) to lie between $10-160^{\circ}$. In Figure 1., we see that our velocities reasonably match the predicted velocity curve obtained with the orbital parameters and mass estimates measured by Pravdo and Shaklan, i.e, the periodicity of 0.744 days, a total mass of the pair of $0.0841 \mathrm{M}_{\text {sol }}$ and a mass for the planet of $6.5 \mathrm{M}_{\text {jup }}$. Additional orbital parameters are provided in Table 5 of Pravdo and Shaklan and within the plot of Figure 2. The observed 
Research, Science and Technology of Brown Dwarfs and Exoplanets

near-infrared velocities and the predicted velocity curve coincide within 1- $\sigma$ uncertainty supporting the astrometry results by Pravdo and Shaklan.

More accurate velocity measurements and a better sampling of the orbital phase are required to precisely constrain the orbital parameters and the individual masses of this planetary system.

\section{References}

[1] S. Pravdo, S. Shaklan, ApJ, 700, 623 (2009)

[2] M. Zapatero Osorio, E. Martin, C. Del Burgo, R. Deshpande, F. Rodler, M.M. Montgomery, A\&A, 505, L5 (2009)

[3] R. Deshpande, E. Martin, M.M. Montgomery, et al. AAS Meeting Abstract, 211, 159.03 (2008)

[4] J. Kirkpatrick, T. Henry, D. Simons, AJ, 109, 797 (1995)

[5] E. Martin, E. Guenther, M. Zapatero Osorio, H. Bouy, R. Wainscoat, 644, L75 (2006)

[6] I. Mclean, E. Becklin, O. Bendiksen, et al., SPIE, 3354, 566 (1998)

[7] M. Zapatero Osorio, E. Martin, H. Bouy, R. Tata, R. Deshpande, ApJ, 647, 1406, (2007)

[8] C. del Burgo, E. Martin, M. Zapatero Osorio, P. Hauschildt, A\&A, 501, 1059 (2009) 